\title{
MODELO FRACTAL DE UNA ERUPCION APLICADO A VOLCANES DENOMINADOS LOG-LOGÍSTICOS: COLIMA, SOUFRIERE Y EREBUS
}

\section{ERUPTION FRACTAL MODEL APPLIED TO LOG-LOGISTIC VOLCANOES: COLIMA, SOUFRIERE AND EREBUS}

\author{
José Brenes-André \\ Red Ciudadana de Estaciones Meteorológicas \\ jbrenes54@gmail.com
}

(Recibido: 19/11/2015; aceptado: 08/04/2016)

\begin{abstract}
The fractal model proposed in Brenes (2013), Brenes \& Alvarado (2013), Brenes (2014) y Brenes (2016) is now expanded to include the empirical log-logistical relationship proposed in Connor et al. (2003) for Soufriere volcano, providing it with a theoretical basis. Such a theoretical derivation suggests a thermo-dynamical view of the eruption process.

Keywords: Balankin, Landau, Brown, Griffith, Glass transition, Soufriere, Colima, Erebus, SFT.

RESUMEN: El modelo fractal propuesto en Brenes (2013), Brenes \& Alvarado (2013), Brenes (2014) y Brenes (2016) es ampliado para deducir una relación log-logística generalizada que puede dar sustento teórico a la relación loglogística empírica propuesta en Connor et al (2003) para el volcán Soufriere. La deducción presentada permite también una interpretación termodinámica del proceso eruptivo.

Palabras clave: Balankin, Landau, Griffith, Transición del vidrio, Soufriere, Colima, Erebus, SFT.
\end{abstract}




\section{ANTECEDENTES}

En Brenes (2013) se presentó la primera aplicación de la SFT (Sequential Fragmentation/ Transport, por su nombre en inglés) a un volcán centroamericano: el Irazú, de Costa Rica, incluyéndose una ampliación para estudiar cada uno de los mecanismos eruptivos de balística, oleadas, saltación y suspensión para aparte, en que se aplicaron los planteamientos de Brown (1989) y Wohletz et al. (1989) cuando la dispersión es negativa. En un trabajo posterior (Brenes \& Alvarado, 2013) se complementó ese análisis con una sistematización de los casos en que la dispersión es positiva, proponiéndose por primera vez que no es un producto de agregación de partículas en vuelo, sino que corresponde a una fragmentación secundaria a la que se le pudo asignar una segunda dimensión fractal. Se incluyó por primera vez una manera de calcular el coeficiente de Hurst asociado a cada sub-población, permitiendo deducir los límites experimentales que Wohletz et al. (1989) encontraron entre actividad magmática y actividad freatomagmática, proponiéndose igualmente un modelo fractal de una erupción que la sistematizó desde el punto de vista fractal por primera vez. Recientemente en Brenes (2014) se amplió el modelo fractal de una erupción para incluir las ideas propuestas por Balankin $(1996,1997)$ a nivel microscópico. De esta manera a partir de consideraciones microscópicas se pudo deducir por primera vez los dos valores universales del coeficiente de Hurst para fractura, y dares una explicación geométrica. En Brenes-André (2016) se incluyó el modelo de Landau de transformaciones de fase de segundo orden, que permitió deducir el modelo de fractura de Griffith, y los tamaños límite deducidos por Balankin (1996,1997), así como sugerir la existencia de fenómenos como el efecto de corrosión por esfuerzos (stress corrotion), y la dilatancia. En este trabajo se amplía ese análisis para incluir la variación en el tiempo, encontrándose de manera natural la ecuación loglogística propuesta empíricamente para analizar la erupción del volcán Soufriere (Connor et al, 2003) y la del volcán Colima (Varley et al., 2006).

\section{APLICACIÓN A ERUPCIONES VOLCÁNICAS}

Dado que los conceptos físicos que se usaran de base para esta derivación no son usualmente conocidos en el campo de la vulcanología, se incluye el siguiente marco de referencia para facilitar su análisis.

Las erupciones de los volcanes suelen analizarse en base a la variación de dos variables termodinámicas: la presión y la temperatura de la lava, lo que desde el punto de vista físico, implica que debe utilizarse la energía libre de Gibbs, que constituye el punto de partida del modelo de Landau.

Un aspecto básico del modelo de transformaciones de segundo orden de Landau es el de rompimiento espontáneo de simetría: se considera que para $\mathrm{T}>\mathrm{T}_{\mathrm{C}}$ el sistema tendrá un mínimo global en $\gamma=0$, en tanto que para $T<T_{C}$ el sistema tendrá un mínimo para $\gamma \neq 0$, que implica la existencia de una fase ordenada de menor simetría.

Para el caso $\beta$ la simetría $\gamma \leftrightarrow-\gamma$ es evidente. Para el caso $\alpha$, sin embargo, encontramos dos expresiones diferentes, una válida para $\gamma<0$, que formalmente tiene la simetría $\gamma \leftrightarrow-\gamma$, y otra para $\gamma$ $>0$ en que hay un término $\gamma^{3}$ que rompe la simetría, y que convierte la transformación de fase en una de primer orden, sin dejar de compartir algunas de las propiedades de los otros casos.

La cristalización de un líquido, cuando su temperatura se baja más allá de una temperatura característica, conocida como punto de solidificación, causa la aparición de estructuras periódicas dentro del conjunto desordenado que es el líquido. Esta cristalización es una transición (de primer orden) acompañada con un rompimiento espontáneo de simetría. Esta es la base del modelo de Landau aplicado a transiciones de segundo orden, como sería el caso de la transición que sufre un pedazo de hierro al ser inmantado. En este trabajo se propone que las erupciones volcánicas son procesos en las que hay transiciones de fase de segundo orden, pues las características de la erupción cambian al cambiar la temperatura o la presión. 
Por experiencia se sabe que en ciertas condiciones se puede bajar la temperatura de un líquido más allá del punto de solidificación, obteniéndose un líquido super-enfriado. Si el líquido en cuestión es sílice $\left(\mathrm{SiO}_{2}\right)$ se observa que al bajar la temperatura del magma éste se solidifica en forma de un vidrio. Es lo que se conoce como Transición al vidrio. Esta transición de fase se produce sin que cambie el calor latente ni en la densidad, lo que implica una transición de segundo orden y, por lo tanto se puede analizar con el modelo de Landau. Esta idea fue desarrollada en Brenes-André (2016) donde se analizó la inclusión del modelo de Landau al modelo fractal de una erupción, en la que en primera aproximación se consideró que la lava era sílice.

La temperatura a la que se da esta transición no es fija pues depende del tratamiento térmico a que fue sometido el magma. Si la aplicación de estas ideas al proceso eruptivo resulta correcta, esta variación podría usarse para obtener más información sobre cómo se afectó la lava en su paso por el conducto volcánico.

Aunque el vidrio que se obtiene tiene propiedades termodinámicas, mecánicas y ópticas específicas, no corresponde a un estado termodinámico estable. Se debe entonces tener extremo cuidado al usar resultados termodinámicos para analizar el proceso de erupción, pues pueden no ser aplicables si son solo válidos en situaciones de equilibrio. Experimentalmente se encuentra que sistemas presuntamente estables pueden ser alterados violentamente y solidificarse instantáneamente si se añade una impureza, o bien el sistema es expuesto a un shock mecánico, que podría tomar la forma de un temblor en el caso de las erupciones volcánicas. Esta podría ser la explicación de porqué un conducto volcánico se cierre durante una erupción.

La transición al vidrio ocurre en substancias que forman líquidos con viscosidades muy altas $\left(10^{5}\right.$ a $\left.10^{7} \mathrm{P}\right)$ que al enfriarse pasan a viscosidades en el ámbito $10^{7}$ a $10^{13} \mathrm{P}$, un fenómeno muy conocido con la lava que interviene en los procesos eruptivos.
La dinámica del proceso de transición tiene un efecto importante en las propiedades de la nueva fase formada. Por ejemplo, si se empieza con una fase líquida, se obtiene una fase cristalina si el magma es enfriado lentamente. Por el contrario, si el magma es enfriado rápidamente (quenched) los mecanismos de crecimiento de núcleos sólidos no actúan, o bien la nucleación de los núcleos de la fase cristalina pueden haberse formado, pero no tienen posibilidad de crecer.

Angell (1995) propuso una clasificación de los vidrios basado en el estudio de la viscosidad de un gran número de líquidos super enfriados. $\mathrm{La}$ viscosidad de los líquidos fuertes (entre ellos $\mathrm{SiO}_{2}$ y $\mathrm{GeO}_{2}$ ) cumplen con la ley de Arrhenius en todo el ámbito de temperatura, y son muy resistentes a degradación por temperatura. Los sistemas con enlaces de hidrógeno son líquidos débiles, y mucho más débiles térmicamente: al ser calentados a temperaturas mayores que $\mathrm{T}_{\mathrm{g}}$, las micro estructuras existentes en el medio tienden a desaparecer. Los líquidos débiles están caracterizados por un gran número de mínimos de energía separados por pequeñas barreras energéticas. Este resultado está de acuerdo con lo esperado en casos de corrosión por esfuerzos (stress corrotion), tal y como se desarrolló en la aplicación de Landau al modelo fractal (Brenes-André, 2016)

\section{ANÁLISIS DINÁMICO}

Según el modelo clásico de Griffith (1923), la energía de una fractura puede escribirse en términos de la semi-longitud $a$ de la fractura:

$$
\begin{aligned}
\mathrm{G}=2 \mathrm{a} \gamma_{\mathrm{o}}-\pi \sigma^{2} \mathrm{a}^{2} / \mathrm{E}=2 \mathrm{a} \gamma_{\mathrm{o}}\left(1-\pi \sigma^{2} \mathrm{a} / 2 \mathrm{E} \gamma_{\mathrm{o}}\right) \\
=4 \gamma_{\mathrm{o}} \mathrm{a}_{\mathrm{c}}\left(\mathrm{a} / 2 \mathrm{a}_{\mathrm{c}}\right)\left(1-\mathrm{a} / 2 \mathrm{a}_{\mathrm{c}}\right)
\end{aligned}
$$

El término $\gamma_{\mathrm{o}}$ es la tensión superficial del material fracturado, que no debe confundirse con la dispersión $\gamma$ obtenida del análisis de las granulometrías de depósitos de ceniza, al utilizar el modelo SFT. El valor a hace mínima la energía G. Dado que $\mathrm{G}$ es la energía de una fractura, el 
término $\gamma_{\mathrm{o}} \mathrm{a}$ podría considerarse como una unidad característica de energía de la fractura. Por lo tanto, adimensionalmente podemos re-escribir la ecuación anterior como

$$
\mathrm{G} / \gamma_{\mathrm{o}} \mathrm{a}_{\mathrm{c}}=4\left(\Omega / \Omega_{\mathrm{eq}}\right)\left(1-\Omega / \Omega_{\mathrm{eq}}\right)
$$

En este punto la expresión $\Omega / \Omega_{\text {eq }}$ es solo una manera de re-escribir la ecuación, pero el análisis que sigue sugiere que la ecuación (2) quizá tenga un significado más profundo.

Partiendo de que los coeficientes $\alpha$ y $\beta$ de Balankin se anulan cuando el sistema alcanza el equilibrio $(\mathrm{dG} / \mathrm{d} \gamma=0)$, en Brenes-André (2016) se propuso que la derivada de la energía de Gibbs $\mathrm{G}$ respecto al parámetro de orden $\gamma$ (la dispersión obtenida a partir de SFT), para el caso del exponente $\alpha$ de Balankin, puede escribirse como:

$$
\begin{aligned}
& \mathrm{dG} / \mathrm{d} \gamma=\mathrm{Q}\left[1-3 \gamma^{2}\right] \gamma /(1+\gamma) \\
& \mathrm{dG} / \mathrm{d} \gamma=\mathrm{Q}\left[1-3 \gamma-3 \gamma^{2}\right] \gamma /(1-\gamma)
\end{aligned}
$$

La ecuación (3), en el caso de que el denominador pueda aproximarse a la unidad, se reducen a la ecuación (1) aplicando la relación $\gamma^{2}=\mathrm{Ka}$, con $\mathrm{K}=\gamma_{\text {eq }}^{2} / \mathrm{a}_{\mathrm{c}}$ (Brenes-André, 2016). La expresión (3) corresponde al caso de dispersión negativa, y la (4) a dispersiones positivas y en ambos casos el coeficiente $\mathrm{Q}<0$ tiene unidades de energía. El término $\gamma$ extra en el numerador se incorporó para lograr que la energía libre $G$ sea mínima cuando $\mathrm{T}>\mathrm{T}_{\mathrm{C}}$ para $\gamma=0$, y mínima para $\gamma \neq 0$ cuando $\mathrm{T}<\mathrm{T}_{\mathrm{C}}$, para poder aplicar el modelo de Landau. Siguiendo a Landau, el término $\mathrm{T}_{\mathrm{C}}$, corresponde a la temperatura a la que sucede la transformación de fase. La inclusión explícita de las temperaturas T y $\mathrm{T}_{\mathrm{C}}$ se desarrolló en Brenes (2016), vía el parámetro adimensional $\tau=\left(\mathrm{T}-\mathrm{T}_{\mathrm{c}}\right) / \mathrm{T}$.

En Brenes-André (2016) a partir de la expresión de la energía libre de Gibbs (G) relacionada con la energía elástica, se puede definir un esfuerzo crítico efectivo $\sigma_{\mathrm{C}}^{1}=\sigma_{\mathrm{C}}\left(\gamma_{\mathrm{eq}} / \gamma\right)$, y siendo la energía elástica proporcional a $\sigma^{2}$ ésta será proporcional a $\left(\gamma / \gamma_{\mathrm{eq}}\right)^{2}$.
La logística aparece entonces por que los efectos competitivos de un stiffening reológico producto de la pérdida de volátiles, que aumenta la presión dinámica en la parte superior del conducto cratérico (Melnik \& Sparks, 1999) como la eventual reducción de la presión debido a la aparición de una red de fracturas (Sparks et al., 2000) pudieron ser expresados en términos del mismo parámetro $\left(\gamma / \gamma_{\mathrm{eq}}\right)^{2}$.

Para el caso del exponente $\beta$ de Balankin, debido a que $\mathrm{H}$ es simétrica respecto a $\gamma$, y no involucra ninguna dimensión fractal, se propuso la expresión

$$
\mathrm{dG} / \mathrm{d} \gamma=\mathrm{Q}(1-2|\gamma|) \gamma /(1-|\gamma|)
$$

incluyéndose de esta manera el principio de von Neumann de que todos los modos compatibles con la simetría deben estar presentes, aunque no necesariamente con el mismo peso. Este análisis inicialmente concentrado en la energía libre $\mathrm{G}$ en función de la dispersión $\gamma$, se ampliará en este trabajo para incluir la variación de la energía libre $\mathrm{G}$ en función del tiempo. Para lograrlo, tal y como es usual, se utilizará la relación $\partial \gamma / \partial \mathrm{t}=-\Gamma \partial \varphi / \partial \gamma$, en la que $\Gamma$ tiene dimensiones inverso de tiempo y $\varphi$ es un potencial en términos del parámetro de orden (la energía de Gibbs $\mathrm{G}$ en nuestro caso).

Con ayuda del gráfico del coeficiente $\beta$ en función de $\gamma, y$ teniendo presente que el origen de la energía de Gibbs es arbitrario, la energía $\mathrm{G}$ puede aproximarse como $\mathrm{Q}\left(\tau \gamma^{2} / 2+\gamma^{4}\right)$ expresión muy similar a la que se obtiene para el coeficiente $\alpha: \mathrm{Q}\left(\tau \gamma^{2} / 2+3 \gamma^{4} / 4\right)$. La similitud es razonable pues una fractura presente en una partícula pequeña (caso $\alpha$ ) tendría la misma energía superficial $\mathrm{Q} \tau \gamma^{2} / 2$ que de estar presente en una partícula más grande (caso $\beta$ ), ya que su creación implica cortar el mismo número de enlaces. La energía elástica almacenada en la partícula, sin embargo, será mayor en una partícula grande $\left(\mathrm{Q} \gamma^{4}\right)$, prácticamente rígida, que en una pequeña (Q $\left.3 \gamma^{4} / 4\right)$, en la que sus paredes externas pueden ceder.

Consideraciones similares sugieren que es razonable que la energía de Gibbs obtenida de la ecuación (4) sea mayor en un factor $-\mathrm{Q} \gamma^{3}>0$, 
producto de las vesícula llenas de gas a las que se ha asociado esta ecuación (Brenes, 2014). En la literatura estos casos se han analizado a partir de la ecuación (1) variando el valor del módulo de Young que se utiliza en los cálculos.

Aplicando la relación $\partial \gamma / \partial \mathrm{t}=-\Gamma \partial \varphi / \partial \gamma$ a los casos de $\alpha$ con dispersión $\gamma$ negativa (ecuación (3), con $\omega=3 / 4$ ), y del exponente $\beta$ (ecuación (5), con $\omega=1)$, con una energía de Gibbs $G=\tau \gamma^{2} / 2$ $+\gamma^{4} \omega$, se deduce

$$
\begin{gathered}
\mathrm{d} \gamma / \mathrm{dt}=-\Gamma_{1} \mathrm{~d}\left(\tau \gamma^{2} / 2+\gamma^{4} \omega\right) / \mathrm{d} \gamma= \\
\Gamma_{1 \tau \mathrm{o} \gamma}\left(1-4 \omega \gamma^{2} / \tau_{\mathrm{o}}\right)
\end{gathered}
$$

El coeficiente $\Gamma_{1}$ es un factor que controla el comportamiento en el tiempo, y $\tau_{\mathrm{o}}=-\tau$ es positivo por tenerse $\mathrm{T}<\mathrm{T}_{\mathrm{C}}$.

La solución de la ecuación (6) es

$$
\gamma^{2}=\left[C \exp \left(-2 \tau_{\mathrm{o}} \Gamma_{1} \mathrm{t}\right)+\left(4 \omega / \tau_{\mathrm{o}}\right)\right]^{-1}
$$

Se ve que $\gamma^{2}$ tiende a $\gamma_{\text {eq }}^{2}=\tau_{\mathrm{o}} / 4 \omega$ conforme el tiempo aumenta, como se había propuesto anteriormente. Por definición $0 \leq \gamma_{\mathrm{eq}} \leq 1$, por lo que la propuesta es válida para temperaturas en el ámbito $\mathrm{T}_{\mathrm{c}}(\omega 4+1)^{-1} \leq \mathrm{T} \leq \mathrm{T}_{\mathrm{c}}$.

Para el caso de una log-logística, la ecuación (5) y su solución (6) se transforman en

$$
\begin{aligned}
& \mathrm{td} \gamma / \mathrm{dt}=\Gamma_{1} \tau_{\mathrm{o}} \gamma\left(1-4 \omega \gamma^{2} / \tau_{\mathrm{o}}\right) \\
& \gamma^{2}=\left[\mathrm{C}\left(-2 \tau_{\mathrm{o}} \Gamma_{1} \mathrm{t}\right)+\left(4 \omega / \tau_{\mathrm{o}}\right)\right]^{-1}
\end{aligned}
$$

A partir de la ecuación (6) y de la relación $\mathrm{d} \gamma^{2} / \mathrm{dt}=2 \gamma \mathrm{d} \gamma / \mathrm{dt}$ obtenemos

$$
\begin{gathered}
\partial(\gamma / \gamma \mathrm{eq})^{2} / \partial \mathrm{t}= \\
-\left(2 \Gamma_{1} / \gamma_{\mathrm{eq}}^{2}\right)\left[4\left(\gamma / \gamma_{\mathrm{eq}}\right)^{2}\left(1-\left(\gamma / \gamma_{\mathrm{eq}}\right)^{2}\right)\right]
\end{gathered}
$$

que es la ecuación de la evolución en el tiempo de la media longitud a de la fractura, pues $\gamma^{2}=$ $\mathrm{Ka}$. En esta formulación el factor 4 se ha singularizado como factor de forma (shape factor) por similitud con la expresión logística de $\mathrm{G} / \gamma_{\mathrm{o}} \mathrm{a}$, ecuación (2). El coeficiente $\left(\gamma_{\mathrm{eq}}^{2} / 2 \Gamma_{1}\right)$ será entonces la unidad natural de tiempo del proceso.
La posibilidad de poder definir un tiempo natural al sistema, permite transformar la ecuación (8) en adimensional.

$$
\begin{aligned}
& \text { RELACIÓN CON } \mathrm{t} d\left(\mathrm{X} / \mathrm{X}_{\mathrm{eq}}\right) / \mathrm{dt}=\mathrm{k}\left(\mathrm{X} / \mathrm{X}_{\mathrm{eq}}\right) \\
& \left(1-\mathrm{X} / \mathrm{X}_{\mathrm{eq}}\right)
\end{aligned}
$$

Los volcanes son sistemas físicos muy complejos que obligan a recurrir a modelos probabilísticos para su estudio, como es el caso de las granulometrías, de las que se puede obtener información valiosa al tratar cada muestra como una suma de varios procesos fractales. En Brenes \& Alvarado (2012) se muestra como un posible evento de mezcla de magmas, así como varios procesos de fragmentación secundaria pudieron ser evidenciados a partir de valores de dispersión positiva aplicando la metodología del SFT.

La propuesta aquí presentada parte de la derivación de la distribución de Weibull (Brown, 1984) a partir del principio de conservación de la masa aplicado a una fragmentación secuencial, obteniéndose que el parámetro $\gamma$ está relacionado con la dimensión fractal del proceso, lo que le da un carácter general. Del modelo de Landau de transición de fases de segundo orden, se ha podido deducir el modelo de Griffith en función de la dispersión $\gamma$. El modelo de fractura de Giffith (ecuación 1) parte de un proceso competitivo entre un proceso superficial, relacionado con el rompimiento de enlaces y la energía superficial $\gamma_{\mathrm{o}}$, y otro volumétrico asociado a la energía elástica que debe invertirse para lograr formar la fractura, al módulo de Young E y el esfuerzo externo $\sigma$. La ecuación (1) basada en la variable a puede transformarse en una ecuación en la variable $\gamma^{2}$ por medio de la relación $\gamma^{2}=$ Ka válida en un ámbito apropiado de la variable a, que incluye el caso particular $\gamma_{\mathrm{c}}{ }^{2}=\mathrm{Ka}_{\mathrm{c}}$, siendo $\mathrm{a}_{\mathrm{c}}$ un valor con una característica especial (en este caso, minimizar la energía libre). Por su carácter fractal, estos procesos se replican a diversos niveles por lo que el parámetro $\mathrm{K}$ no cambia. De esta manera, es posible re-escribir la ecuación (1) en función de una semilongitud crítica $a_{c}$, que aunque es una relación 
multiplicativa de propiedades del material $\left(\gamma_{0} \mathrm{y}\right.$ E), y de condiciones externas $(\sigma)$, tal dependencia se elimina si se trabaja con la relación $\mathrm{a} / \mathrm{a}_{\mathrm{c}}$.

La posibilidad de formular un modelo de Landau utilizando la dispersión $\gamma$ como parámetro de orden permite que este procedimiento pueda repetirse en cada caso en que se pueda establecer una relación $\gamma^{2}=\mathrm{K} \Omega$ con una propiedad $\Omega$ dada, $\mathrm{y}$ un factor $\mathrm{K}$ apropiado, dando lugar a la ecuación (2). Se propone entonces que la relación 4 (X/ Xeq) (1- $\left.\mathrm{X} / \mathrm{X}_{\mathrm{eq}}\right)$ aquí derivada es una expresión genérica que deben seguir las propiedades observables relacionadas con el proceso de fractura, y que puede aplicarse a algunos de los fenómenos utilizados por Connor et al (2003) para sustentar su propuesta empírica.

Como se señala en Connor et al. (2003), en Voight $(1988,1989)$ y en Cornelius \& Voight (1994) se propone que propiedades observables $(\Omega)$ tales como la deformación, el inclinamiento, la energía sísmica (todas relacionadas al proceso de fractura) pueden modelarse por la ecuación $\mathrm{d} \Omega / \mathrm{dt}=\mathrm{A} \mathrm{t}^{\mathrm{k}}$. Esta propuesta deriva a su vez de ideas desarrolladas por Weibull (1951) para obtener la tasa de falla en materiales.

El proceso eruptivo a través de un conducto fuerza a que el proceso de fragmentación del magma se de exclusivamente en la capa en contacto con la atmósfera por lo que, siguiendo a Shore et al (1992), se propone que el proceso se enlentece apreciablemente dando lugar a un crecimiento logarítmico. Por tanto, la ecuación logística debe convertirse en una log-logística

$$
\operatorname{td}\left(X / X_{e q}\right) / d t=k\left(X / X_{e q}\right)\left(1-X / X_{e q}\right)
$$

que es la ecuación (10) propuesta empíricamente en Connor et al. (2003) y que aplicaría a los casos del exponente de Balankin tanto $\beta$, como al caso $\alpha$, con dispersión negativa. El coeficiente de forma (shape factor) de 4, que se deduce en la ecuación (1), se ha transformado en un parámetro k para poder incluir modificaciones a la propuesta inicial de Griffith. Esta es una posición similar a la adoptada por Voight $(1988,1989)$ en su formulación del Método de predicción de falla de materiales (FFM, Materials Failure Forcasting Method), basado a su vez en las ideas de Weibull (1951).
Conceptualmente este análisis tiene una similitud con lo propuesto por Lu et al (2005) a partir de la variación en el tiempo de una relación no lineal de esfuerzo $\sigma$ vs deformación $\varepsilon$. Como lo indica el título de su trabajo, tratan el caso de una falla catastrófica que se muestra por medio de una reducción de la dimensión fractal, punto medular en nuestra propuesta (Brenes, 2014) para incluir el modelo de Balankin.

Experimentalmente se encuentra que los datos de eventos explosivos del volcán Colima (Mayo 2002) se ajustaron mejor con una distribución log-logística (Varley et al., 2006), lo que también se encuentra para los datos obtenidos en 1998 para el volcán Karymsky, Para los volcanes Soufriere (Connor et al., 2003) y el volcán Colima se encontraron factores de forma cercanos a 4 , lo que se interpretó como indicador de una similitud entre ellos.

Varley et al. (2000) llaman igualmente la atención a datos obtenidos en el periodo Octubre 1997 a Julio 1998 para el Volcan Erebus, que sugieren la existencia de dos poblaciones diferentes, una de ellas asociada a un factor de forma de 1,70 sujeto a diversas observaciones. Sin embargo, se apunta igualmente que el mejor ajuste se dio con una log-logística.

La incorporación del modelo de Landau, basada en la conservación de la energía, permite no solo deducir la ecuación original de Griffith, sino también incluir, vía $\tau_{\mathrm{o}}$, efectos como la temperatura y la entropía lo que sugiere que la expresión deducida en el presente trabajo tiene una aplicación bastante general. Así, los efectos de las vesículas, no incluidas en la formulación de Griffith, se incorporan vía el término cúbico $\gamma^{3}$, y los esfuerzos externos se reflejan en la fuerza conjugada al parámetro de orden $\gamma$.

Aunque la aplicación de $\partial \gamma / \partial \mathrm{t}=-\Gamma \partial \varphi / \partial \gamma$ sugiere que el potencial $\varphi$ controla la dinámica de un sistema (ecuación 9), el carácter de teoría de campo medio del modelo de Landau posiblemente impide incluir casos en que una observación más detallada puede llevar a reconocer patrones de corta duración (Denlinger \& Hoblitt, 1999). Los patrones a una escala temporal más grande, por su parte, requieren de análisis estadísticos para evidenciarlos, usando el registro temporal 
de erupciones como una serie temporal en que los datos de intervalos temporales de reposo (repose time) se ajustan a una función de densidad de probabilidad según un modelo determinado. De esta manera, si el ajuste es bueno se puede obtener información relevante sobre el proceso que genera el evento.

Las ideas desarrolladas en Connor et al (2006), sugieren que la solución log-logística aquí presentada provee un mejor ajuste a los periodos de quietud que la distribución exponencial. La distinción es importante pues si el tiempo entre el último evento y el siguiente es realmente loglogístico (dependiendo de que se llegue a entender por evento) la tasa de riesgo sería constante, y así se tendría a mano una información preventiva.

\section{CONCLUSIONES}

En áreas como la física del estado sólido es común encontrar que en procesos analizados con ayuda del modelo de transformaciones de segundo orden de Landau, se recurra a la relación $\partial \gamma / \partial \mathrm{t}$ $=-\Gamma \partial \varphi / \partial \gamma$ para estudiar la variación temporal del parámetro de orden. En el caso de las erupciones volcánicas la aplicación de $\partial \gamma / \partial t=-\Gamma \partial \varphi / \partial \gamma$ sería entonces razonable en casos que involucren magma explosivo, no así en los casos de magma efusivo. La relación logística se pudo derivar al incorporar los coeficientes derivados por Balankin al modelo de Landau. Por ello, la propuesta empírica de Voight, $(1988,89)$, Cornelius \& Voight (1994), y Connor et al (2003), y su eventual confirmación empírica afianza tanto la inclusión de Landau como la escogencia del parámetro de dispersión $\gamma$ (obtenido a partir de la SFT) como el parámetro de orden, reforzando la propuesta de un modelo fractal. Por último, la conversión de la relación logística en una log-logística, al proponer que el proceso de fragmentación tendría un carácter predominantemente superficial, a partir de la analogía con procesos de estado sólido, se refleja en la fragmentación que ocurre en el interface lava-atmósfera.

El análisis aquí presentado puede ampliarse para incluir procesos netamente termodinámicos. Dado que la relación $\partial \gamma / \partial \mathrm{t}=-\Gamma \partial \varphi / \partial \gamma$ aplica a procesos adiabáticos, es posible que los valores de $\mathrm{k}$ menores a 4 puedan reflejar erupciones más cercanas a procesos isotérmicos, en los que el proceso de fractura involucra no solo la interface lava-atmósfera.

Esta idea está en línea con los resultados de Fokin et al. (1981) en disilicato de litio $\left(\mathrm{Li}_{2} \mathrm{O}-\right.$ $2 \mathrm{SiO}_{2}$ ) según los cuales en un vidrio que sufre una exposición isotérmica previa a un proceso de nucleación no-estacionario, hay primero un crecimiento exponencial en el tiempo de los centros de nucleación, que eventualmente se transforma en un crecimiento lineal. Llama la atención que si el número de centros de nucleación por unidad de volumen $\mathrm{N}$ fuese inversamente proporcional a $\gamma^{2}$ (cumple con $\gamma^{2}=\mathrm{K} \Omega$ ), el crecimiento exponencial (ecuación 5), y el posterior crecimiento lineal (ecuación 5a) coincidirían con los resultados presentados en Fokin et al. (1981)

Esto sugiere la posibilidad de que, previo a la erupción, una porción apreciable del magma esté a una misma temperatura (exposición isotérmica previa) con su alrededor y, en algún momento, la parte superior empiece a nuclearse adiabáticamente conforme vaya dándose el proceso de erupción. El factor de forma (shape factor) k sería entonces una relación entre el tiempo característico de formación de fracturas en el magma, y el tiempo característico del proceso de difusión de esas fracturas en el magma.

\section{REFERENCIAS BIBLIOGRÁFICAS}

ANGELL, C. A., 1995: Formation of glasses from liquids and biopolymers.- Science, 267: 1924-1935.

BRENES, J., 2013: Aplicación de la teoría de fragmentación/transporte secuencial a los depósitos de las erupciones 1723 y $1963-$ 65 del Irazú, Costa Rica. Caso dispersión negativa.- Rev. Geol. Amér. Central, 48: 63-85.

BRENES, J., \& ALVARADO, G. E., 2013: Aplicación de la teoría de fragmentación/ transporte secuencial a los depósitos de 
las erupciones 1723 y 1963-65 del Irazú, Costa Rica. Caso dispersión positiva y modelo fractal.- Rev. Geol. Amér. Central, 48: 87-98.

BRENES, J., 2014: Aplicación del modelo de fractura fractal de Balankin al modelo fractal de actividad volcánica. - Rev. Geol. Amér. Central, 50: 83-98.

BRENES-ANDRE, J., 2016: Incorporación del modelo de Landau al modelo fractal de una erupción.- Rev. Geol. Amér. Central, 54: $125-142$.

CONNOR, C. B., SPARKS, R. S. J., MASON, R. M., BONADONNA, C. \& YOUNG, S. R., 2003.- Exploring links between physical and probabilistic models of volcanic eruptions: The Soufrie're Hills Volcano, Montserrat.- Geophys R. Lett. 30, 1701, DOI:10.1029/2003GL017384, 2003

CONNOR, C. B., MCBIRNEY, A. R. \& FURLAN, C., 2006: What is the probability of explosive eruption at long-dormant volcano?.- En: MADER, H. M., COLES, S. G., CONNOR, C. B., CONNOR, L. J. (eds): Statistics in Volcanology. Special Publications of International Association of Volcanology and Chemistry of the Earth's Interior 1 (IAVCEI).- Geological Soc. London, London: 39-46.

CORNELIUS, R. R. \& VOIGHT, B., 1994-. Seismological aspects of the 1989-1990 eruption of Redoubt volcno, Alaska: The materials failure forecast method (FFM) with RSAM and SSAM seismic data.- J. Volcan. Geotherm. Res. 62; 469-498.

DENLINGER, R. P. \& HOBLITT, R. P. 1999. Cyclic eruptive behaviour of silicic volcanoes.- Geology, 27: 459-462.
FOKIN, V. M., KALINIA, A. M. M. \& FILIPOVICH, V. N. 1981- Nucleation in silicate glasses and effect of preliminary heat treatment on it.- J. Cryst. Growth, 52: 115 .

GUNN, L. S.; BLAKE, S.; JONES, M. C. \& RYMER, H., 2014: Forecasting the duration of volcanic eruptions: an empirical probabilistic model.- Bull Volcanol 76:780, DOI 10.1007/s00445-013-0780-8

LU, Y-C., MAIY, Y-W. \& XIEZ, H., 2005: A sudden drop of fractal dimension: a likely precursor of catastrophic failure in disordered media.- Philosophical Magazine Letters, 85: 33-40.

MELNIK, O. \& SPARKS, R. S. J., 1999: Nonlinear dynamics of lava dome extrusion.Nature, 402: 37-41.

SHORE, J. D., HOLZER, M., \& SETHNA, J. P., 1992: Logarithmically slow domain growth in nonrandom frustrated systems: Ising models with competing interactions. Phys. Rev. B, 46: 11376-404.

SPARKS, R. S. J., MURPHY, M. D., LEJEUNE, A. M., WATTS, R. B., BARCLAY \& YOUNG, S. R., 2000: Control on the emplacement of the andesite dome of the Soufriere Hills Volcano, Monserrat, by degassing-induced crystallization.- Tera Nova, 12: 14-20.

VARLEY, N., JOHNSON, J., RUIZ, M., REYES, G. \& MARTIN, K., 2006: Applying Statistical Analysis to Understanding the Dynamics of Volcanic Explosions.En MADER, H. M., COLES, S. G., CONNOR, C. B. \& CONNOR, L. J. (eds): Statistics in Volcanology. Special Publications of International Association 
of Volcanology and Chemistry of the Earth's Interior 1 (IAVCEI). Geol. Soc. London, London: 57-76.

VOIGHT, B., 1988: A method for prediction of volcanic eruptions.- Nature, 332: 125-130.
VOIGHT, B., 1989: A relation to describe ratedependent material failure.- Science, 243: 200-203.

WEIBULL, W., 1951: A statistical distribution of wide applicability.- J. Appl. Mech, 18: 293-297. 
